\title{
A ferret model of COPD-related chronic bronchitis
}

\author{
S. Vamsee Raju, ${ }^{1}$ Hyunki Kim, ${ }^{2}$ Stephen A. Byzek, ${ }^{1}$ Li Ping Tang, ${ }^{1}$ John E. Trombley, ${ }^{1}$ \\ Patricia Jackson, ${ }^{1,3}$ Lawrence Rasmussen, ${ }^{1}$ J. Michael Wells, ${ }^{1,3,4}$ Emily Falk Libby, ${ }^{1,4}$ Erik Dohm, ${ }^{5}$ \\ Lindy Winter, ${ }^{6}$ Sharon L. Samuel, ${ }^{2}$ Kurt R. Zinn, ${ }^{2}$ J. Edwin Blalock,, ${ }^{1,3,4}$ Trenton R. Schoeb ${ }^{4,7}$ \\ Mark T. Dransfield, ${ }^{1,3,4}$ and Steven M. Rowe ${ }^{1,3,4,6,8}$ \\ 'Department of Medicine, ${ }^{2}$ Department of Radiology, ${ }^{3} \mathrm{U} A B$ Lung Health Center, and ${ }^{4} \mathrm{Gregory}$ Fleming James Cystic \\ Fibrosis Research Center, University of Alabama at Birmingham, Birmingham, Alabama, USA. ${ }^{5}$ Animal Resources \\ Program, Birmingham, Alabama, USA. ${ }^{6}$ Department of Pediatrics, 'Department of Genetics, and ${ }^{8}$ Department of Cell, \\ Developmental, and Integrative Biology, University of Alabama at Birmingham, Birmingham, Alabama, USA.
}

Chronic obstructive pulmonary disease (COPD) is the third leading cause of death in the US. The majority of COPD patients have symptoms of chronic bronchitis, which lacks specific therapies. A major impediment to therapeutic development has been the absence of animal models that recapitulate key clinical and pathologic features of human disease. Ferrets are well suited for the investigation of the significance of respiratory diseases, given prior data indicating similarities to human airway physiology and submucosal gland distribution. Here, we exposed ferrets to chronic cigarette smoke and found them to approximate complex clinical features of human COPD. Unlike mice, which develop solely emphysema, smoke-exposed ferrets exhibited markedly higher numbers of early-morning spontaneous coughs and sporadic infectious exacerbations as well as a higher level of airway obstruction accompanied by goblet cell metaplasia/hyperplasia and increased mucus expression in small airways, indicative of chronic bronchitis and bronchiolitis. Overall, we demonstrate the first COPD animal model exhibiting clinical and pathologic features of chronic bronchitis to our knowledge, providing a key advance that will greatly facilitate the preclinical development of novel treatments for this disease.

Conflict of interest: The authors have declared that no conflict of interest exists.

Submitted: March 16, 2016 Accepted: August 18, 2016 Published: September 22, 2016

Reference information: JCI Insight. 2016;1(15):e87536. doi:10.1172/jci.insight.87536.

\section{Introduction}

Chronic obstructive lung disease (COPD) has recently surpassed stroke as the third leading cause of death in the US (1), and it is observed with increasing incidence worldwide. Although genetic factors also contribute to COPD etiology, cigarette smoking and chronic exposure to inhaled irritants, such as pollutants, particulate matter, and chemical fumes, pose the main risks (2). Unfortunately, current treatment options for this disease are limited. No pharmacologic treatments for COPD alter its natural history, and there are no effective treatments for chronic cough and sputum production, which are characteristic of the chronic bronchitis phenotype and are among the most clinically troublesome COPD manifestations. As such, management is confined to smoking cessation, oxygen therapy, and symptomatic treatment, primarily using bronchodilators.

Two classical phenotypes of COPD are emphysema and chronic bronchitis. Recent understanding of these disease phenotypes suggests far more overlap in terms of underlying mechanisms and presentation than what was previously recognized in the literature $(3,4)$. Chronic bronchitis patients traditionally exhibit pathologic features, including goblet cell hyperplasia, mucin hyperexpression, and mucus accumulation (5), which lead to impaired mucus clearance (6-8), chronic bacterial colonization, and persistent neutrophilic airway inflammation (9-13). A relatively high incidence of bronchiectasis (14) and association with lung function decline (15) are further indicative of COPD progression. Airway mucus obstruction in chronic bronchitis is associated with excess morbidity and mortality and is a major contributor to airflow limitation $(12,16)$.

To date, the absence of suitable animal models has severely limited progress in evaluating the mechanistic basis of COPD and in developing new, more efficacious therapies. Most prominently, smoke-exposed rodents and genetically modified mice do not exhibit mucus retention, despite the presence of severe 
emphysema (17). Noting the demonstration of spontaneous bronchitis and bronchopneumonia $(18,19)$ in a recently developed ferret model of cystic fibrosis via knockout of cystic fibrosis transmembrane conductance regulator (CFTR), which has also been observed to be dysfunctional in COPD patients who are active or former smokers $(20,21)$, we hypothesized that ferrets may be more suitable for the study of COPD pathophysiology. Prior reports indicating impaired mucus transport following smoke exposure of ferrets provided further support to model COPD in this species (22). Thus, we sought to develop an animal model of cigarette smoke-induced chronic bronchitis and found that ferrets exposed to chronic smoke developed defining clinical and pathologic characteristics of the disease. This research tool will help advance our understanding of mechanisms underlying COPD pathogenesis and provide an improved preclinical model for evaluating novel COPD therapies.

\section{Results}

Evidence of chronic bronchitis in cigarette smoke-exposed ferrets. Chronic bronchitis is clinically defined by the presence of chronic productive cough. To develop a useful animal model of smoke-induced chronic bronchitis, normal ferrets were exposed to cigarette smoke for 6 months. Following a brief training period to acclimate the ferrets, fully grown normal males and females were exposed to 60 minutes of smoke from $3 \mathrm{R} 4 \mathrm{~F}$ research cigarettes $(200 \mu \mathrm{g} / 1$ of total particulate matter) twice daily using a custom-designed nose-only exposure system coupled with an automated smoke generator (Supplemental Figure 1; supplemental material available online with this article; doi:10.1172/jci.insight.87536DS1). After 3 months of cigarette smoke exposure, ferrets began to develop a noticeable spontaneous cough that worsened over time. To quantify this, we recorded early-morning coughs at 6 months of smoke exposure using whole-body plethysmography combined with audio and video confirmation in conscious free-moving ferrets. Recordings were collected for 10-minute sessions at least 15 hours after the most recent smoke exposure. A representative cough is shown in Supplemental Video 1. Abrupt changes in the respiratory cycle of smoke-exposed ferrets are apparent by whole-body plethysmography tracings and are accompanied by audible cough (Figure 1A). Early-morning cough frequency was significantly elevated in smoke-exposed ferrets (Figure 1B). Together, these data indicate that smoke-exposed ferrets exhibit clinical evidence of chronic bronchitis even in the absence of direct irritation resulting from acute smoke exposure.

Chronic mucus hypersecretion and goblet cell metaplasia/hyperplasia. To assess pathologic evidence of chronic mucus hypersecretion, the pathologic correlate to chronic bronchitis, we examined ferrets upon necropsy performed 6 months following cigarette smoke exposure. This time period is equivalent to the human life expectancy projected after approximately 10 pack-years of smoking, the guideline-recommended threshold for a clinical diagnosis of COPD (23). All morphometric analyses of ferret airways were conducted by a pathologist blinded to exposure assignment for two lobes (upper and lower left) from each ferret; 98 periodic acid-Schiff-positive (PAS-positive) airway sections randomly chosen from each treatment group were available for analysis. PAS staining revealed clear evidence of goblet cell metaplasia/hyperplasia in medium and small airways (Figure 2, A and B). Submucosal gland hyperplasia also was evident (Figure 2A), reminiscent of increased Reid index in humans with chronic bronchitis. Epithelial height was approximately 20\% increased over air controls (Figure 2, C and D), even when controlled for airway caliber (Figure 2E), indicating epithelial remodeling and hypertrophy. Mucus expression measured by PAS-positive goblet cell area, normalized by the size of the airway lumen to account for cell variation due to airway diameter, was $60 \%(0.042 \% \pm 0.025 \%$ smoke vs. $0.025 \% \pm 0.013 \%$ air control; $P=0.06)$ higher in smoke-exposed airways over control airways (Figure $2 \mathrm{~F}$ ). Individual ferrets exhibited heterogeneity in this parameter, reflecting the patchy nature of bronchitis. Goblet cell distribution analyzed by airway size quintile indicated that increased mucus was most evident in the smallest airways analyzed (Figure 2G), as thought to occur in humans (12). Muc5b and Muc5ac staining was greater in smoke-exposed ferrets (Supplemental Figure 2) but was not conducive to quantitation due to patchy staining. These results in ferrets were distinct from results from experiments involving prolonged exposure of cigarette smoke to mice, which showed minimal evidence of goblet cell metaplasia/hyperplasia or mucus obstruction (Supplemental Figure 3), consistent with extensive prior experience with smoke-exposed mouse models $(21,24)$.

Chronic bronchitis and infectious exacerbations in cigarette smoke-exposed ferrets. Compromised host defense observed in patients with COPD fosters an airway environment that facilitates growth of inhaled and aspirated bacteria, setting the stage for pathologic infections $(25,26)$. Over the 6-month period of cigarette smoke exposure, 2 of 12 smoke-exposed ferrets developed fatal respiratory infection, with fever, respiratory 
A

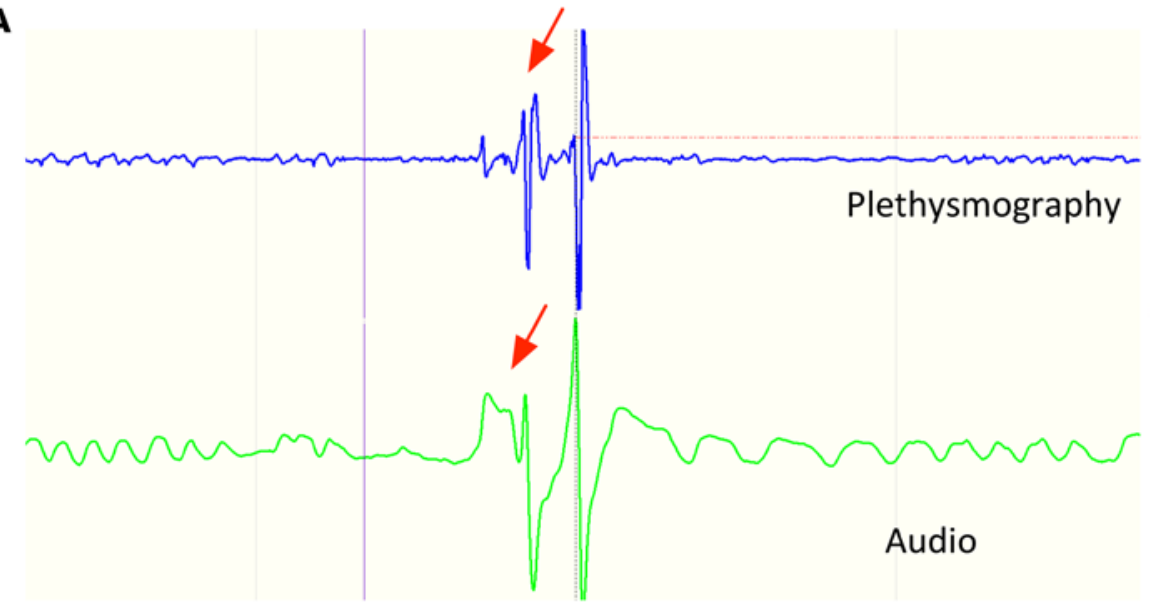

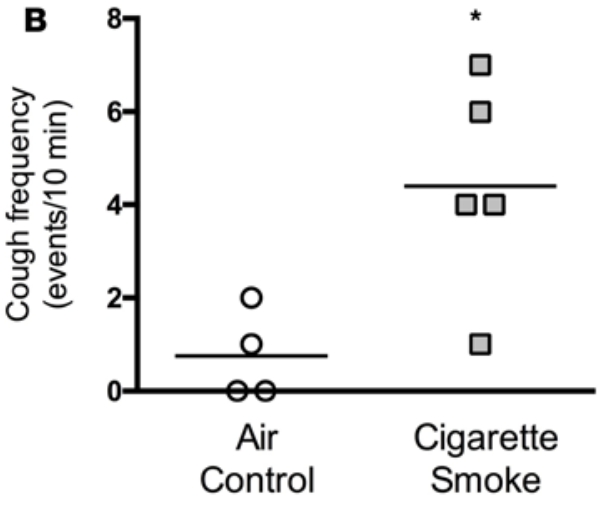

Figure 1. Evidence of chronic cough in a ferret model of COPD. (A) Representative plethysmograph (blue) and audio (green) tracings of a ferret inside an integrated cough monitor. The typical change in respiratory cycle and audio recording during a cough event is designated by the red arrows. See Supplemental Video 1 for a representative video. (B) Summary of verified early-morning, unstimulated coughs in ferrets exposed to 6 months of either cigarette smoke or room air. $n=4-6 /$ group. ${ }^{*} P<0.05$.

distress, reduced feeding, weight loss, and physical inactivity necessitating euthanasia and necropsy (Figure $3 \mathrm{~A})$. Even among the ferrets that did not exhibit clinical signs of infection, smoke exposure caused weight loss (Figure 3B), reminiscent of the lower BMI observed among COPD patients $(27,28)$. Gross examination revealed mucus occlusion of larger airways in ferrets with bronchitis exacerbations (Figure 3C), and microbial cultures of lung specimens identified Staphylococcus xylosus and Staphylococcus schleiferi, pathogenic respiratory bacteria native to ferrets (29-31). Histologic analysis revealed mucus plaques plastered to the airway surface (Figure 3D) and focal areas of respiratory bronchiolitis and peribronchial pneumonia (Figure $3 \mathrm{E}$ ), characteristics of airway infection that were not observed in controls.

Airway obstruction in cigarette smoke-exposed ferrets. Airway obstruction is associated with COPD morbidity and mortality, even in patients who do not chronically expectorate mucus $(12,16)$. To assess for the presence of small airway obstruction in the ferret model, we examined airway function using forced oscillometry to assess respiratory mechanics in intubated and sedated ferrets. Inspiratory capacity, a sensitive marker of resting hyperinflation correlated with COPD mortality (32), was significantly reduced in smoke-exposed ferrets (79.5 $\pm 9.4 \mathrm{ml}$ smoke vs. $85.9 \pm 5.9 \mathrm{ml}$ air control; $P<0.05$; Figure $4 \mathrm{~A})$. Because inspiratory capacity can be decreased due to either increased small airway obstruction or loss of elastic recoil from emphysema, we examined other respiratory parameters to distinguish these possibilities. Reduced inspiratory capacity was associated with a $43 \%$ increase $(P<0.05)$ in Newtonian airway resistance, which primarily reflects the conducting airways (Figure 4B). In contrast, neither tissue damping (Figure 4C) nor quasistatic compliance (Figure 4D), each a marker of alveolar resistance and elastic recoil, respectively, were significantly altered. In total, these data provide functional evidence of airway obstruction in smoke-exposed ferrets.

Emphysema in cigarette smoke-exposed ferrets. In addition to demonstrating features of chronic bronchitis, a unique feature as compared with other animal models, smoke-exposed ferrets exhibited some histological features of emphysema, as seen in humans and mice. Evidence of emphysema in smoke-exposed ferrets included a pathologic increase in airspace structure, as shown by alveolar enlargement compared to controls (Figure 5, A and B), as reflected in a 15\% increase in mean linear intercept (Figure 5E), a quantitative measure of emphysema that is increased by a similar magnitude in humans with COPD but is lower than that seen in mice $(33,34)$. Pathologic analysis demonstrated peribronchial and interstitial neutrophilic inflammation (Figure 5, C and D) that was accompanied by increased neutrophil counts in bronchoalveolar lavage fluid (Figure 5F). Smoke exposure had a minimal effect on lung compliance, suggesting a mild emphysema as opposed to overt chronic bronchitis at this stage of disease (Figure 4D), compatible with the duration of smoking. Taken together, these studies provide evidence that 6 months of cigarette smoke exposure in ferrets induces classic features of mild-to-moderate COPD, including features of both chronic bronchitis and emphysema. 
A

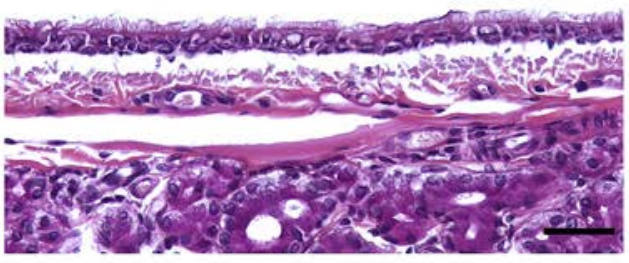

Cigarette Smoke

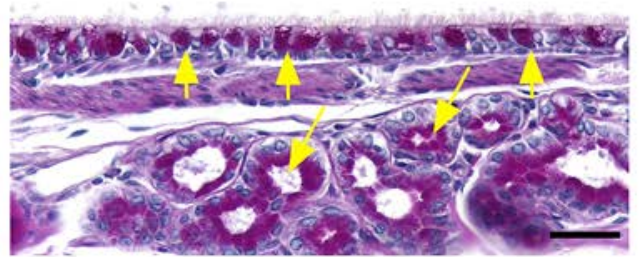

D

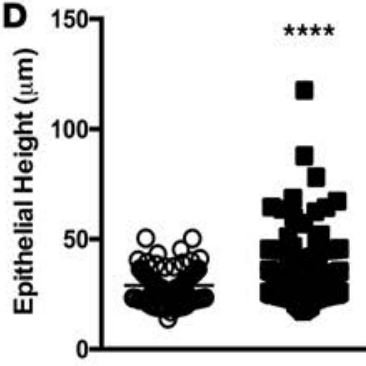

Air Cigarette Control Smoke

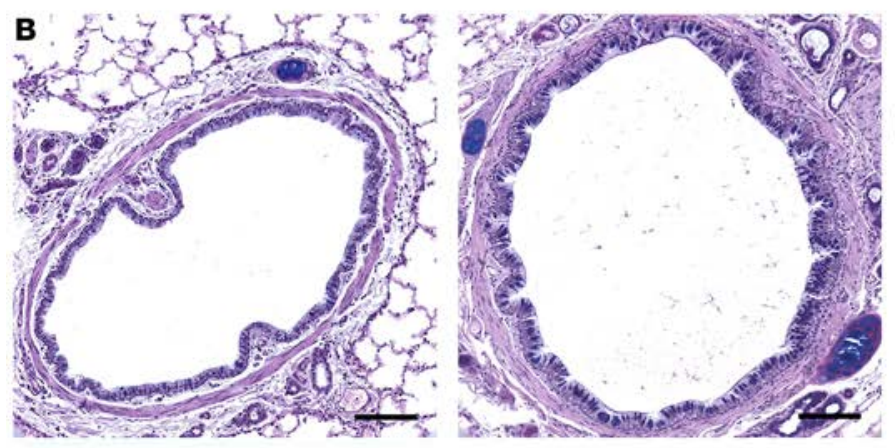

C

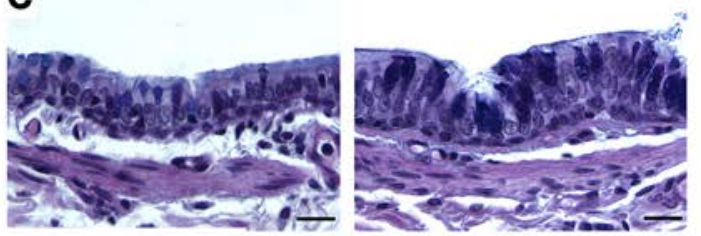

E

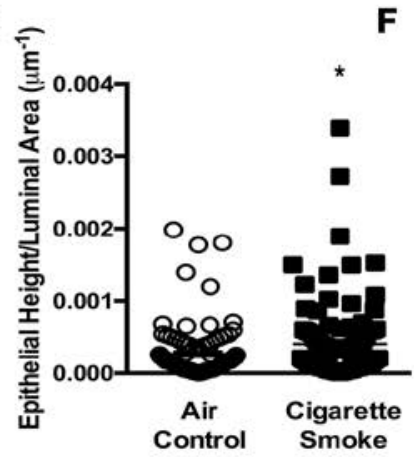

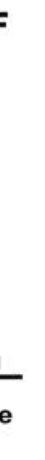

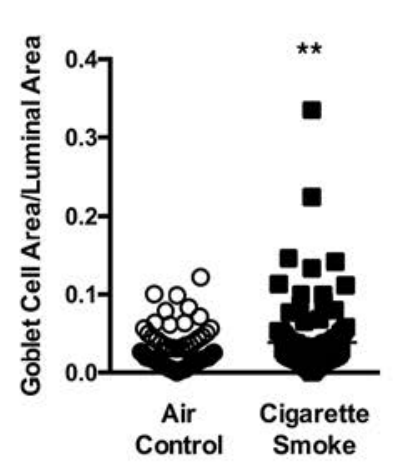

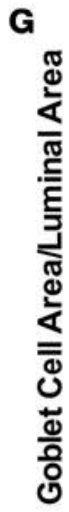

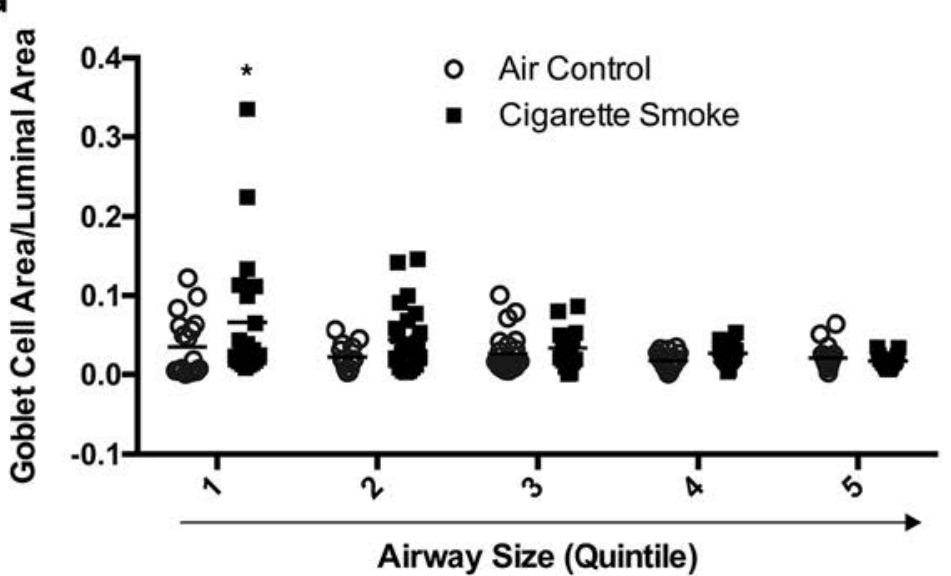

Figure 2. Histopathologic evidence of chronic mucus hypersecretion and goblet cell hyperplasia in a ferret model of COPD. (A) Mucus-expressing goblet cells and submucosal glands (yellow arrows) were stained with PAS (magenta) in tracheal sections of ferrets exposed to room air or cigarette smoke for 6 months. Scale bar: $34 \mu \mathrm{m}$. (B) Similarly, lung sections from the same ferrets were stained with AB-PAS to highlight mucus-expressing cells (deep blue). Scale bar: $110 \mu \mathrm{m}$. (C) The same staining as in B demonstrating goblet cell hyperplasia and increased epithelial cell height. Scale bar: $17 \mu \mathrm{m}$. (D and E) Quantitative analyses demonstrating epithelial hyperplasia, as measured by increased epithelial cell height (D) controlled for luminal area (E). (F) Goblet cell area controlled for luminal area in cigarette smoke-exposed ferrets. (G) Goblet cell area controlled for luminal area expressed as a function of the airway luminal (inner) diameter quintile (diameter; quintile 1: 116-293 $\mu \mathrm{m}$, quintile 2: 294-415 $\mu \mathrm{m}$, quintile 3: 424-535 $\mu \mathrm{m}$, quintile 4: 537-793 $\mu \mathrm{m}$, and quintile 5: $810-2,651 \mu \mathrm{m}) . n=8$ /group. ${ }^{*} P<0.05,{ }^{* *} P<0.01,{ }^{* * *} P<0.0001$.

\section{Discussion}

In the US, nearly 15 million individuals suffer from COPD, generating over $\$ 30$ billion in annual healthcare costs $(1,2)$. Over $60 \%$ of COPD patients have cough and sputum production characteristic of chronic bronchitis, which is independently associated with rate of lung function decline and death and is without effective treatment $(12,35,36)$, rendering this COPD phenotype particularly problematic. Limitations of currently available animal models of COPD have posed a major barrier to gaining mechanistic insight into and developing novel treatment strategies for this disease. We have tackled this critical shortcoming by developing a cigarette exposure system for ferrets, an animal model that we show exhibits clinical and pathological features of bronchitis similar to those observed in humans, and coupling 

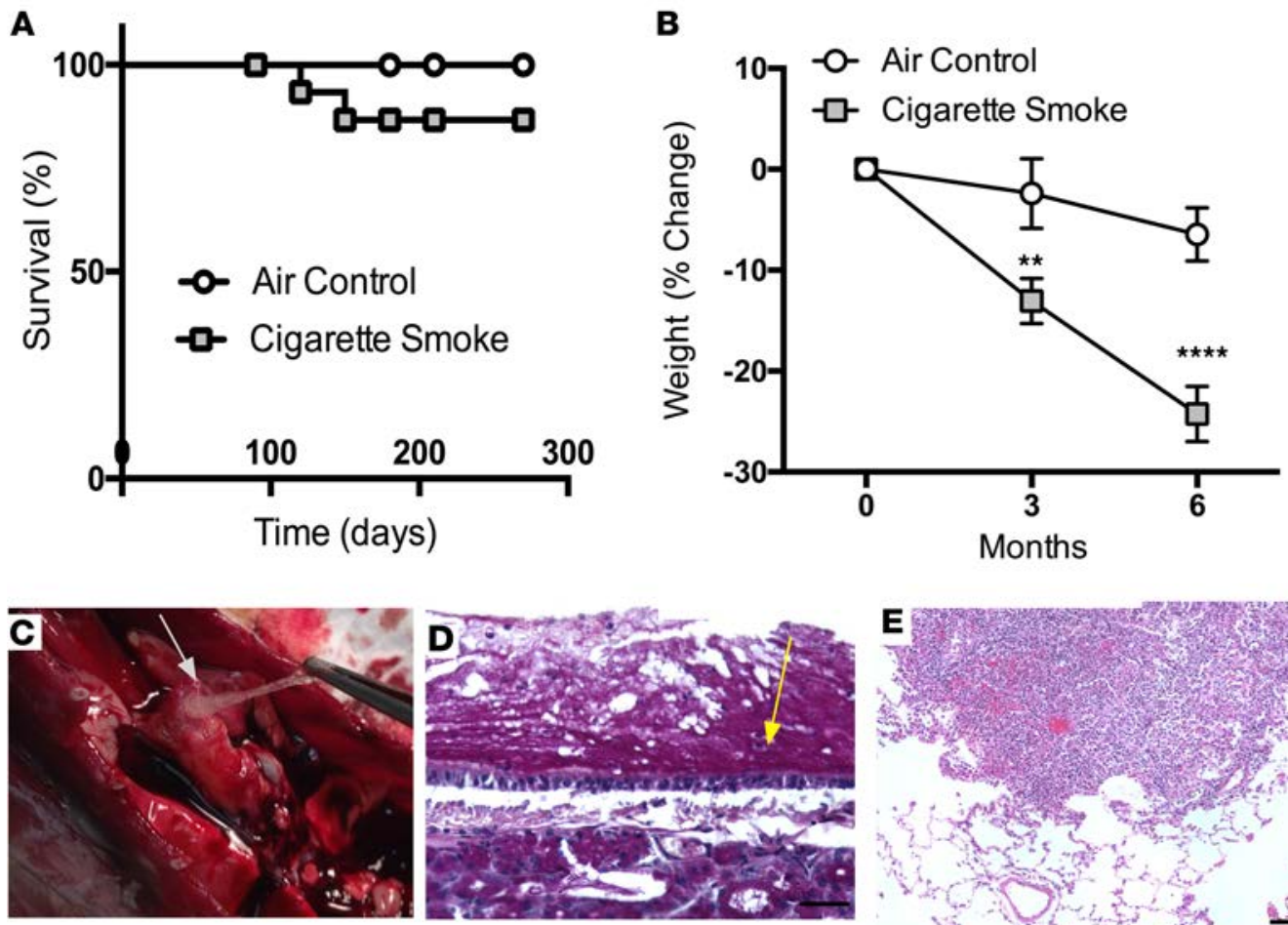

Figure 3. Chronic bronchitis exacerbations in cigarette smoke-exposed ferrets. (A) Kaplan-Meier survival curve of ferrets exposed to cigarette smoke or room air for 6 months. (B) Body weight changes observed in cigarette smokeexposed ferrets compared with air controls over 6 months of exposure. (C) Image (original magnification, $\times 2$ ) capturing evidence of mucus obstruction in a large airway shown in a smoke-exposed ferret that suffered from spontaneous lung infection with Staphylococcus xylosus and Staphylococcus schleiferi. (D and E) Histopathologic examination confirmed mucus accumulation in the trachea by PAS staining (D, yellow arrow; scale bar: $135 \mu \mathrm{m})$ and inflammatory foci $(\mathbf{E}$; scale bar: $135 \mu \mathrm{m}$ ) in lung highlighted by $\mathrm{H} \& \mathrm{E}$ staining. $n=8$ /group. ${ }^{* *} P<0.01,{ }^{* * *} P<0.0001$.

this with a number of clinically relevant assays. After a sufficient duration and intensity of exposure, smoke-exposed ferrets exhibit cough and airway obstruction as well as pathologic evidence of chronic mucus hypersecretion, the cardinal features of the human condition.

Clinically, chronic bronchitis is diagnosed when a patient presents with cough and mucus on most days for 3 months during 2 consecutive years when other lung conditions have been reasonably eliminated as a cause. To our knowledge, this is the first report of recapitulating early-morning cough in a COPD animal model without any use of stimulatory agents such as capsaicin or citric acid. In COPD patients, cough frequency is highest in the morning, especially in those patients with severe disease $(37,38)$. In addition, early-morning cough can also be used to predict exacerbation risk, rescue inhaler usage and response, ability to carryout normal daily activities, and overall health-related quality of life (39-41). Thus, smoke-exposed ferrets serve as an effective laboratory tool to evaluate pathogenic events leading up to chronic cough, elucidate mechanisms, and test emerging interventions.

The link between chronic bronchitis symptoms and airway obstruction is not always straightforward, and hence, it is critical to elucidate the mechanistic nuances underlying these manifestations. For example, mucin overproduction and mucus obstruction of small airways occurs even in COPD patients with emphysema and documented airflow obstruction but without evidence of chronic bronchitis (12). Conversely, some patients with chronic mucus expectoration do not have airflow obstruction (42). Our data are consistent with the complexities of the human condition, as COPD ferrets exhibit clear evidence of chronic bronchitis (cough, conducting airway abnormalities) and bronchiolitis (increased mucus expression in small airways), but the changes in airway obstruction are more variable. We suspect that goblet cell metaplasia/ hyperplasia, hypertrophied epithelium, and mucus accumulations are responsible for diminished inspiratory capacity and increased Newtonian resistance, a readout primarily of the large and conducting airways, 


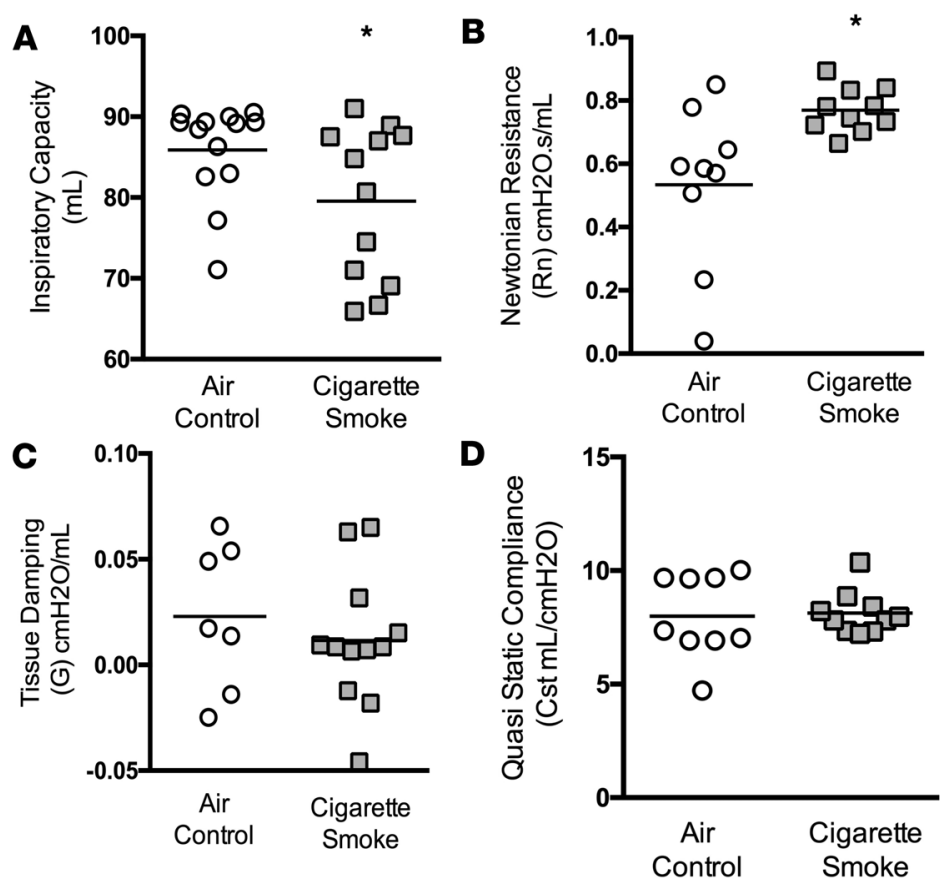

Figure 4. Lung function analyses in a ferret model of COPD. Functional estimate of airway obstruction in ferrets exposed to cigarette smoke or their corresponding air controls was carried out using a forced oscillometry-based system. (A) Compared with control ferrets, ferrets that underwent chronic smoke exposure for 6 months exhibited significantly diminished inspiratory capacity, a sensitive marker of airway obstruction. (B) Newtonian resistance, a measure of airway resistance of the conducting airways, was elevated in COPD ferrets. (C and D) Tissue damping (C), a measure of tissue elastance, and quasistatic compliance

(D) were not significantly affected by smoking. $n=$ 9-12/group. ${ }^{*} P<0.05$.

among smoke-exposed ferrets. In contrast, while increased mucus expression of the small airways was clearly significant upon histopathologic analysis, measures of small airway resistance (i.e., tissue damping) were not elevated in smoke-exposed ferrets. While changes in tissue damping may have been masked by less dramatic changes in the alveolar spaces, this may also reflect the disparity between histopathology and physiology apparent in humans and suggest the need for other sensitive endpoints to quantify the relative contribution of small airway disease to lung obstruction.

Although features of chronic bronchitis are prominent in the ferret model, emphysema also clearly occurs, mirroring emerging clinical data that are challenging the widely accepted dichotomy that there are two distinct COPD phenotypes - emphysema and chronic bronchitis $(3,43,44)$. Smoke-exposed ferrets exhibit an increased mean linear intercept that is proportionate to alveolar enlargement seen in humans (33) (Figure 5), reflecting the overlap of these classically distinct phenotypes. We suspect that the contribution of emphysema to airway obstruction in smoke-exposed ferrets is relatively modest, given minimal alterations in lung compliance and more prominent mucosal obstruction (Figure 4); this may be due to the relatively mild stage of disease at this stage of the model (i.e., 10 pack-years based on life expectancy of ferrets). The fact that bronchitis remains prominent is compatible with the high incidence of bronchitis in smokers without obstruction or mild (i.e., GOLD stage I and II) disease $(42,45)$. Since ferrets are not inbred, both genetic and environmental triggers - such as the presence of infection, intensity of smoking, and the effect of smoking cessation - for disease progression could be evaluated in future studies to help understand risk factors and causative features associated with progression from bronchitis alone to overt COPD.

COPD exacerbations are a major source of morbidity, mortality, and healthcare costs (46-49). Among the prominent features of the ferret model are respiratory exacerbations that occur spontaneously and bear striking similarities to human COPD exacerbations in regards to their severity, microbial features, and histopathological findings. We demonstrate that ferrets exposed to cigarette smoke develop sporadic respiratory exacerbations characterized by reduced physical activity, severe mucus retention, and overt bacterial infection (as opposed to subclinical colonization) centered around the airways (Figure 3). These findings set the stage for future studies to uncover the importance of impaired mucus clearance and abnormal mucosal defense in predisposing patients with COPD to recurrent infections, an aspect of the pathogenesis of COPD exacerbations that has not been emphasized in recent years but is of renewed interest, given their contribution to disease morbidity and progression $(50,51)$. The pathologic organisms isolated to date are native to ferrets, suggesting that defects in epithelial innate defense create a permissive host environment for pathologic colonization. The same is thought to occur in humans, in which altered pathogenicity has been reported in the setting of COPD exacerbations (46). Like the ferrets in our studies, patients with chron- 
A

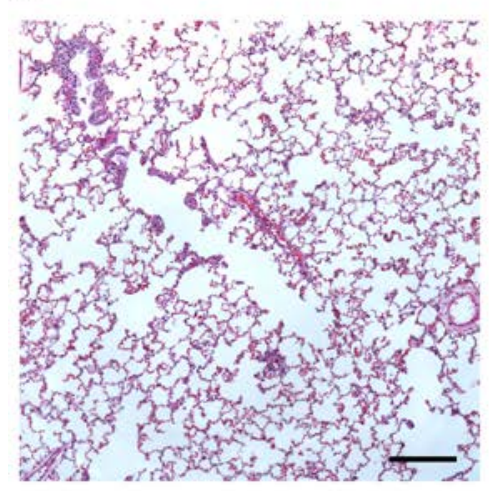

B

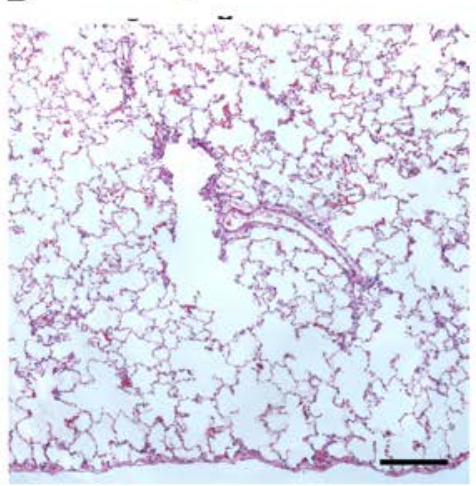

C

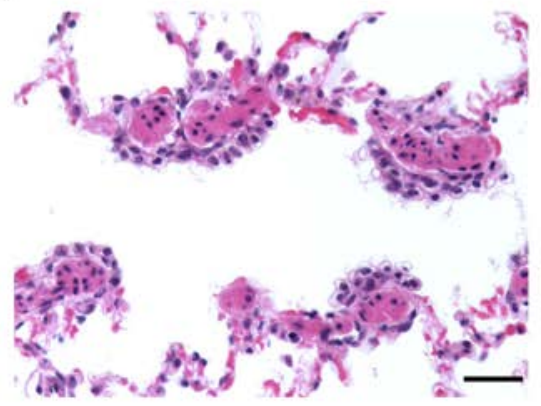

D

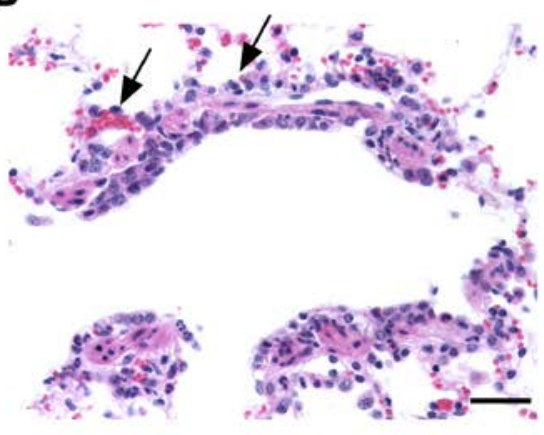

E

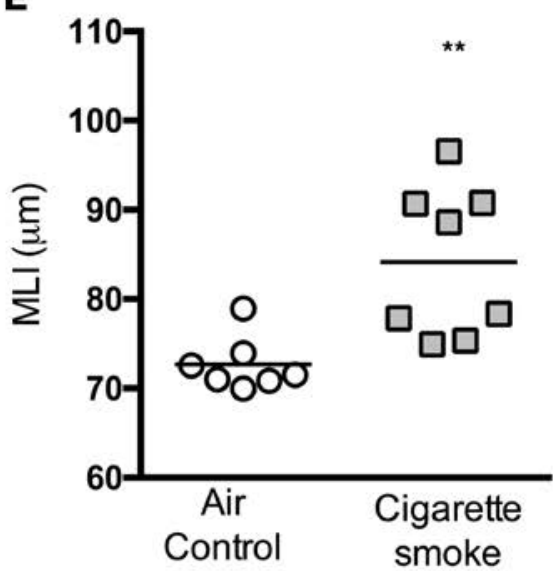

$F$
Fo
0
$\times$
00
$\frac{0}{2}$
0
0
$\frac{1}{5}$
2
2
$\frac{1}{2}$
0

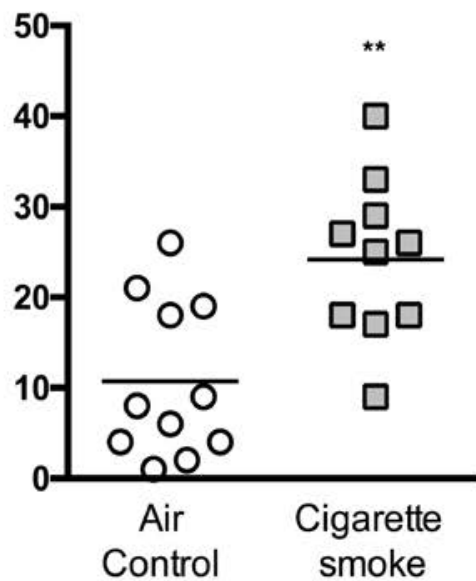

Figure 5. Emphysema and neutrophilic inflammation in smoke-exposed ferrets. (A and B) Histopathologic evidence of emphysematous airspace enlargement shown in representative lung sections of ferrets exposed to cigarette smoke and their corresponding air controls. Scale bar: $220 \mu \mathrm{m}$. (C and D) H\&E-stained section of respiratory bronchioles, indicating an influx of neutrophils (black arrows) in ferrets exposed to cigarette smoke for 6 months compared with air controls. Scale bar: $34 \mu \mathrm{m}$. (E) Summary of mean linear intercept (MLI), a marker of alveolar enlargement, in ferrets exposed to cigarette smoke or air controls for 6 months. (F) Summary of mean neutrophil counts observed in bronchoalveolar lavage fluids in cigarette smoke-exposed ferrets and their air control counterparts. $n=8-12$ /group. ${ }^{*} P<0.01$.

ic bronchitis and minimal airway obstruction also develop exacerbations that cause significant morbidity disproportionate to the severity of the underlying disease and that are significant contributors to disease progression $(52,53)$. The clinical features of these exacerbations warrant further study and could yield potential interventions to mitigate their incidence and/or severity.

In summary, we have developed an animal model of smoke-induced chronic bronchitis, which recapitulates human COPD by exhibiting features of emphysema, bronchiolitis, and airway obstruction. This model presents exciting opportunities to evaluate in detail the pathogenesis of chronic bronchitis and airway infection; the role of sporadic exacerbations in altering the natural history of COPD; and the potential to interrupt disease progression by testing specific therapeutic interventions.

\section{Methods}

Cigarette smoke exposure in ferrets. Age- and gender-matched wild-type ferrets (Mustela putorius furo, females, $0.6-0.8 \mathrm{~kg}$ in body weight; males, $1.2-2.0 \mathrm{~kg}$ in body weight) were procured from Marshall BioResources and randomly assigned to whole cigarette smoke exposure or air control groups. Following a brief period of acclimatization and training, ferrets were restrained in customized male and female nose-only exposure tubes, with a 24-port plenum connected to mainstream smoke output. Ferrets were exposed to 60 minutes of smoke from 3R $4 \mathrm{~F}$ research cigarettes, twice daily for 6 months. Cigarette smoke was generated by an 
automated cigarette smoke generator (SCIREQ, InExpose model), with a 24-cigarette autoloading carousel. An in-line gas analyzer for oxygen, carbon monoxide, and particulate matter provided real-time estimates of cigarette smoke intensity and ensured accurate and safe exposure of animals. Animals were monitored continuously, and analytics demonstrated particulate matter $(200 \mu \mathrm{g} / 1$ of total particulate matter) and CO levels $(\sim 1 \%-3 \%)$ typical of other animal exposure systems.

Standardized anesthesia and endotracheal intubation. For lung function measurements, ferrets were anesthetized with a formulation containing dexmedetomidine $(0.08-0.2 \mathrm{mg} / \mathrm{kg}, \mathrm{IM})$ and ketamine $(2.5-5 \mathrm{mg} /$ $\mathrm{kg}, \mathrm{IM})$. Ophthalmic petroleum jelly was applied to the eyes once sedation was achieved to minimize drying. The animals were placed on a heated operating table until recovery. Pulse oximetry was used to monitor oxygenation and the depth of anesthesia. Supplemental oxygen was provided, if needed. Atipamezole $(5.0 \mathrm{mg} / \mathrm{ml})$ was administered as a reversal agent upon completion of the procedures, followed by a brief monitoring period. Ferrets were intubated with a 3.0-mm cuffed endotracheal tube by an expert operator using a laryngoscope equipped with a Miller blade (size 0). Endotracheal intubation was confirmed with a pediatric end-tidal $\mathrm{CO}_{2}$ detector.

Cough monitoring. Unrestrained ferrets were analyzed using a cough monitoring system (EMKA Technologies) capable of performing simultaneous video, acoustic, and plethysmographic recordings. Cough analysis included acoustic and visual recordings compared with those of plethysmography, indicated by abrupt deep inhalation and exhalation characteristic of cough. Early-morning coughs (between 7 and 9 $\mathrm{AM}$ ) were recorded at least 16 hours following the last smoke exposure, without any stimulation with inhaled irritants. Frequency of cough was monitored once the animal was acquainted with the chamber. Cough data were acquired for 10 minutes per session.

Lung function measurements. Spirometric lung function in ferrets was characterized using the FlexiVent mechanical ventilator (SCIREQ), using module 5 according to the manufacturer's instructions. Ferrets were sedated, then endotracheally intubated and connected to the in-line ventilator for multiple forced maneuvers. Following acquisition of baseline parameters, anesthetized ferrets received succinylcholine $(2-5 \mathrm{mg} / \mathrm{kg}$, i.v.) to relax voluntary breathing for up to 10 minutes and improve signal acquisition for respiratory parameters, including resistance, compliance, elastance, inspiratory capacity, and pressure-volume loops. Ferrets remained on ventilator support until they recovered from sedation and were able to breathe spontaneously.

Necropsy and histopathologic analysis. Anesthetized ferrets were euthanized by exsanguination when in clinical distress or at the end of the study. Separate lung lobes were designated for histopathology, microbiome/microbiology, and biochemical analysis of inflammation. Bronchoalveolar lavage fluids were collected with $10 \mathrm{ml}$ of sterile saline from right lungs, except from the cordate lobe that was cryopreserved for protein expression analyses. Left lungs were inflated by instilling $70 \%$ alcoholic formalin to a pressure of $25 \mathrm{~cm}$ of water and fixed in excess formalin for a minimum of 48 hours before being sectioned and embedded in paraffin. Tissue sections were examined by a board-certified veterinary pathologist for evidence of inflammatory cell infiltrate, mucus stasis, alveolar enlargement, and additional histopathologic changes in direct comparison to controls. A veterinary pathologist remained blinded to treatment assignments during these analyses and quantitative morphometry.

Reagents. All chemicals used in this study were obtained from Sigma-Aldrich.

Statistics. Descriptive statistics (mean, SD, and SEM) were compared using 2-sided Student's $t$ test or 2-way ANOVA, as appropriate. Post-hoc tests for multiple comparisons following ANOVA were calculated using Fisher's least significant difference only if ANOVA was significant. All statistical tests were 2-sided and were performed at a 5\% significance level (i.e., $\alpha=0.05$ ) using GraphPad Prism software. Error bars designate SEM unless indicated otherwise.

Study approval. All animal protocols used in this work were reviewed and approved by the University of Alabama at Birmingham Institutional Animal Care and Use Committee.

\section{Author contributions}

SVR, MTD, and SMR conceived of the experiments; SVR, HK, SAB, LPT, JET, PJ, LR, JMW, ED, SLS, and TRS conducted the research; LW, KRZ, JEB, and TRS provided reagents and techniques; SVR, HK, SAB, LPT, JET, LR, JMW, TRS, MTD, and SMR analyzed the data; SVR, MTD, EFL, and SMR wrote the manuscript; SVR and SMR supervised the project. All authors had an opportunity to edit the manuscript and approved of its submission. 


\section{Acknowledgments}

This work was funded by NIH R01HL105487 (to SMR), NIH P30 DK072482 (to SMR), NIH R01 HL07783 (to JEB), NIH R01 HL110950 (to JEB), NIH R01 HL114439 (to JEB), and the Forest Research Institute. SVR was supported by the American Lung Association (RG-305752) and the Flight Attendant Medical Research Association (YFA130008). FlexiVent was utilized as a component of the University of Alabama at Birmingham Program in Protease and Matrix Biology (principle investigator, Amit Gaggar) and supported, in part, by the following grants: NIH R01 HL102371 and Veterans Administration I01BX001756.

Address correspondence to: Steven M. Rowe, MCLM 7056, 1918 University Boulevard, Birmingham, Alabama 35294-0006, USA. Phone: 205.934.9640; E-mail: smrowe@uab.edu.

1. National Heart, Lung, and Blood Institute. Unpublished Tabulations of the National Health Interview Survey, 2010. http:// www.cdc.gov/nchs/nhis/nhis_2010_data_release.htm. Updated December 14, 2012. Accessed September 21, 2016.

2. Ford ES, Murphy LB, Khavjou O, Giles WH, Holt JB, Croft JB. Total and state-specific medical and absenteeism costs of COPD among adults aged $\geq 18$ years in the United States for 2010 and projections through 2020. Chest. 2015;147(1):31-45.

3. Hogg JC. Pathophysiology of airflow limitation in chronic obstructive pulmonary disease. Lancet. 2004;364(9435):709-721.

4. Rabe KF, Beghe B, Luppi F, Fabbri LM. Update in chronic obstructive pulmonary disease 2006. Am J Respir Crit Care Med. 2007;175(12):1222-1232.

5. Saetta M, et al. Goblet cell hyperplasia and epithelial inflammation in peripheral airways of smokers with both symptoms of chronic bronchitis and chronic airflow limitation. Am J Respir Crit Care Med. 2000;161(3 Pt 1):1016-1021.

6. Morgan L, et al. Scintigraphic measurement of tracheal mucus velocity in vivo. Eur Respir J. 2004;23(4):518-522.

7. Brown JS, Zeman KL, Bennett WD. Ultrafine particle deposition and clearance in the healthy and obstructed lung. Am J Respir Crit Care Med. 2002;166(9):1240-1247.

8. Moller W, et al. Deposition, retention, and translocation of ultrafine particles from the central airways and lung periphery. Am $J$ Respir Crit Care Med. 2008;177(4):426-432.

9. Rowe SM, Miller S, Sorscher EJ. Cystic fibrosis. N Engl J Med. 2005;352(19):1992-2001.

10. Rab A, Rowe SM, Raju SV, Bebok Z, Matalon S, Collawn JF. Cigarette smoke and CFTR: implications in the pathogenesis of COPD. Am J Physiol Lung Cell Mol Physiol. 2013;305(8):L530-L541.

11. Raju SV, Solomon GM, Dransfield MT, Rowe SM. Acquired cystic fibrosis transmembrane conductance regulator dysfunction in chronic bronchitis and other diseases of mucus clearance. Clin Chest Med. 2016;37(1):147-158.

12. Hogg JC, et al. The nature of small-airway obstruction in chronic obstructive pulmonary disease. $N$ Engl J Med. 2004;350(26):2645-2653.

13. Wells JM, et al. An aberrant leukotriene A4 hydrolase-proline-glycine-proline pathway in the pathogenesis of chronic obstructive pulmonary disease. Am J Respir Crit Care Med. 2014;190(1):51-61.

14. Martinez-Garcia MA, et al. Factors associated with bronchiectasis in patients with COPD. Chest. 2011;140(5):1130-1137.

15. Allinson JP, Hardy R, Donaldson GC, Shaheen SO, Kuh D, Wedzicha JA. The presence of chronic mucus hypersecretion across adult life in relation to chronic obstructive pulmonary disease development. Am J Respir Crit Care Med. 2016;193(6):662-672.

16. Hogg JC, et al. Survival after lung volume reduction in chronic obstructive pulmonary disease: insights from small airway pathology. Am J Respir Crit Care Med. 2007;176(5):454-459.

17. Groneberg DA, Chung KF. Models of chronic obstructive pulmonary disease. Respir Res. 2004;5:18

18. Sun X, et al. Lung phenotype of juvenile and adult cystic fibrosis transmembrane conductance regulator-knockout ferrets. $A m J$ Respir Cell Mol Biol. 2014;50(3):502-512.

19. Sun X, et al. Disease phenotype of a ferret CFTR-knockout model of cystic fibrosis. J Clin Invest. 2010;120(9):3149-3160.

20. Dransfield MT, et al. Acquired cystic fibrosis transmembrane conductance regulator dysfunction in the lower airways in COPD. Chest. 2013;144(2):498-506

21. Raju SV, et al. Cigarette smoke induces systemic defects in cystic fibrosis transmembrane conductance regulator function. Am $J$ Respir Crit Care Med. 2013;188(11):1321-1330.

22. Rasmussen RE, Mannix RC, Oldham MJ, Phalen RF. Effects of nitrogen dioxide on respiratory tract clearance in the ferret. $J$ Toxicol Environ Health. 1994;41(1):109-120.

23. Vestbo J, et al. Global strategy for the diagnosis, management, and prevention of chronic obstructive pulmonary disease: GOLD executive summary. Am J Respir Crit Care Med. 2013;187(4):347-365.

24. Hautamaki RD, Kobayashi DK, Senior RM, Shapiro SD. Requirement for macrophage elastase for cigarette smoke-induced emphysema in mice. Science. 1997;277(5334):2002-2004.

25. Erb-Downward JR, et al. Analysis of the lung microbiome in the "healthy" smoker and in COPD. PLoS One. 2011;6(2):e16384.

26. Sze MA, Hogg JC, Sin DD. Bacterial microbiome of lungs in COPD. Int J Chron Obstruct Pulmon Dis. 2014;9:229-238.

27. Vestbo J, et al. Body mass, fat-free body mass, and prognosis in patients with chronic obstructive pulmonary disease from a random population sample: findings from the Copenhagen City Heart Study. Am J Respir Crit Care Med. 2006;173(1):79-83.

28. Celli BR, et al. The body-mass index, airflow obstruction, dyspnea, and exercise capacity index in chronic obstructive pulmonary disease. N Engl J Med. 2004;350(10):1005-1012.

29. Jean-Pierre H, Darbas H, Jean-Roussenq A, Boyer G. Pathogenicity in two cases of Staphylococcus schleiferi, a recently described species. J Clin Microbiol. 1989;27(9):2110-2111.

30. Latorre M, Rojo PM, Unzaga MJ, Cisterna R. Staphylococcus schleiferi: a new opportunistic pathogen. Clin Infect Dis. 1993;16(4):589-590.

31. Bingel SA. Pathology of a mouse model of x-linked chronic granulomatous disease. Contemp Top Lab Anim Sci. 2002;41(5):33-38. 
32. Tantucci C, et al. Inspiratory capacity predicts mortality in patients with chronic obstructive pulmonary disease. Respir Med. 2008;102(4):613-619.

33. Thurlbeck WM. Overview of the pathology of pulmonary emphysema in the human. Clin Lab Med. 1984;4(3):539-559.

34. Robbesom AA, et al. Aberrant fibrillin-1 expression in early emphysematous human lung: a proposed predisposition for emphysema. Mod Pathol. 2008;21(3):297-307.

35. Vestbo J, Prescott E, Lange P. Association of chronic mucus hypersecretion with FEV1 decline and chronic obstructive pulmonary disease morbidity. Copenhagen City Heart Study Group. Am J Respir Crit Care Med. 1996;153(5):1530-1535.

36. Vestbo J. Epidemiological studies in mucus hypersecretion. Novartis Found Symp. 2002;248:3-12; discussion 12.

37. Partridge MR, Karlsson N, Small IR. Patient insight into the impact of chronic obstructive pulmonary disease in the morning: an internet survey. Curr Med Res Opin. 2009;25(8):2043-2048.

38. Kim YJ, et al. Patient's perception of symptoms related to morning activity in chronic obstructive pulmonary disease: the SYMBOL Study. Korean J Intern Med. 2012;27(4):426-435.

39. Partridge MR, Miravitlles M, Ståhl E, Karlsson N, Svensson K, Welte T. Development and validation of the Capacity of Daily Living during the Morning questionnaire and the Global Chest Symptoms Questionnaire in COPD. Eur Respir J. 2010;36(1):96-104

40. Small M, Broomfield S, Pollard R, Fermer S. Impact of morning symptoms experienced by COPD patients on exacerbation risk, rescue inhaler usage and normal daily activities. Eur Respir J. 2012;40(56): P3476.

41. Roche N, Chavannes NH, Miravitlles M. COPD symptoms in the morning: impact, evaluation and management. Respir Res. 2013;14:112.

42. Martinez $\mathrm{CH}$, et al. The clinical impact of non-obstructive chronic bronchitis in current and former smokers. Respir Med. 2014;108(3):491-499.

43. Hogg JC. State of the art. Bronchiolitis in chronic obstructive pulmonary disease. Proc Am Thorac Soc. 2006;3(6):489-493.

44. Friedlander AL, Lynch D, Dyar LA, Bowler RP. Phenotypes of chronic obstructive pulmonary disease. COPD. 2007;4(4):355-384.

45. Kim V, et al. The chronic bronchitic phenotype of COPD: an analysis of the COPDGene Study. Chest. 2011;140(3):626-633

46. Sethi S, Murphy TF. Infection in the pathogenesis and course of chronic obstructive pulmonary disease. $N$ Engl J Med. 2008;359(22):2355-2365.

47. Sethi S, Evans N, Grant BJ, Murphy TF. New strains of bacteria and exacerbations of chronic obstructive pulmonary disease. $N$ Engl J Med. 2002;347(7):465-471.

48. Huang YJ, Sethi S, Murphy T, Nariya S, Boushey HA, Lynch SV. Airway microbiome dynamics in exacerbations of chronic obstructive pulmonary disease. J Clin Microbiol. 2014;52(8):2813-2823.

49. Larsen JM, et al. Divergent pro-inflammatory profile of human dendritic cells in response to commensal and pathogenic bacteria associated with the airway microbiota. PLoS ONE. 2012;7(2):e31976.

50. Donaldson GC, et al. Impact of Prolonged Exacerbation Recovery in Chronic Obstructive Pulmonary Disease. Am J Respir Crit Care Med. 2015;192(8):943-950.

51. Donaldson GC, Seemungal TA, Bhowmik A, Wedzicha JA. Relationship between exacerbation frequency and lung function decline in chronic obstructive pulmonary disease. Thorax. 2002;57(10):847-852.

52. Regan EA, et al. Clinical and Radiologic Disease in Smokers With Normal Spirometry. JAMA Intern Med. 2015;175(9):1539-1549.

53. Bowler RP, et al. Prediction of acute respiratory disease in current and former smokers with and without COPD. Chest. 2014;146(4):941-950. 